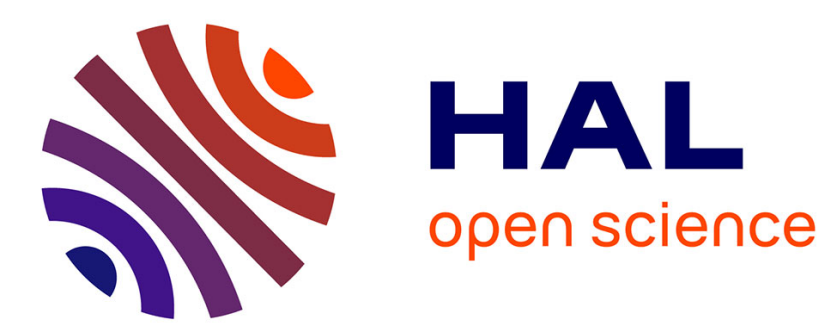

\title{
Equilibrium, games, and pricing in transportation and telecommunication networks
}

\author{
Eitan Altman, Laura Wynter
}

\section{To cite this version:}

Eitan Altman, Laura Wynter. Equilibrium, games, and pricing in transportation and telecommunication networks. [Research Report] RR-4632, INRIA. 2002. inria-00071953

\section{HAL Id: inria-00071953 \\ https://hal.inria.fr/inria-00071953}

Submitted on 23 May 2006

HAL is a multi-disciplinary open access archive for the deposit and dissemination of scientific research documents, whether they are published or not. The documents may come from teaching and research institutions in France or abroad, or from public or private research centers.
L'archive ouverte pluridisciplinaire HAL, est destinée au dépôt et à la diffusion de documents scientifiques de niveau recherche, publiés ou non, émanant des établissements d'enseignement et de recherche français ou étrangers, des laboratoires publics ou privés. 
INSTITUT NATIONAL DE RECHERCHE EN INFORMATIQUE ET EN AUTOMATIQUE

\section{Equilibrium, games, and pricing in transportation and telecommunication networks}

Eitan Altman and Laura Wynter

$\mathbf{N}^{\circ} 4632$

Novembre 2002

THÈMES 1 et 4 



\title{
Equilibrium, games, and pricing in transportation and telecommunication networks*
}

\author{
Eitan Altman ${ }^{\dagger}$ and Laura Wynter ${ }^{\ddagger}$ \\ Thèmes 1 et 4 - Réseaux et systèmes - Simulation et optimisation \\ de systèmes complexes \\ Projets MISTRAL et METALAU
}

Rapport de recherche $\mathrm{n}^{\circ} 4632$ - Novembre 2002 - 17 pages

\begin{abstract}
Network equilibrium models that have traditionally been used for transportation planning have penetrated in recent years to other scientific fields. These models have recently been introduced in the telecommunication networks literature, as well as in the field of game theory. Researchers in the latter fields are not always aware of the very rich literature on equilibrium models outside of their application area. On the other hand, researchers that have used network equilibrium models in transportation may not be aware of new application areas of their tools. The aim of this paper is to present some central research issues and tools in network equilibria and pricing that could bring closer the three mentioned research communities.
\end{abstract}

Key-words: Nash equilibrium, Wardrop equilibrium, traffic assignment

\footnotetext{
* This work was partially supported by a research contract with France Telecom R\& D No. 101 E 0162

$\dagger$ INRIA, Sophia Antipolis

$\ddagger$ INRIA, Rocquencourt and PRISM, Université de Versailles
} 


\section{Equilibres, jeux, et tarification des réseaux de transports et de télécommunications ${ }^{\S}$}

Résumé : L'étude d'équilibre du trafic dans les réseaux de transports a récemment été adaptée aux réseaux de télécommunications. Ce type d'équilibre, différent de celui de Nash, commence à être étudié aussi dans la théorie de jeux. L'objet de ce papier est de présenter les résultats de recherche obtenus par les communautés du transport, des télécommunications, et de la théorie des jeux.

Mots-clés : Equilibre de Nash, équilibre de Wardrop, affectation de trafic

$\S$ Ce rapport a été financé en partie par un contrat de recherche de France Télécom R \& D, No. 101 E 0162 


\section{Introduction}

Determining the equilibrium state of a traffic network has been a preoccupation of transport planners for nearly half a century. Underlying this preoccupation is the assumption that road traffic will naturally arrange itself in an equilibrium flow, under steady state conditions. In this context, predictions of future traffic flow patterns that would follow any changes to the network or demand levels rely upon an accurate representation of the traffic equilibrium.

Except for recent years, telecommunication network flow models have not made use of the notion of network equilibrium, as traffic networks do. Indeed, while road traffic is highly individualized, each driver making his or her own route choice decision, telecommunication networks are much more centralized.

Recently, however, equilibrium models have started to emerge in telecommunication networks. This is due to two main reasons. First, the deregulation and privatization of large telecom companies introduced competitive and decentralized behavior among telecom operators. Secondly, a new concept of networks has been developed and deployed with a large success, in which most of the intelligence lies at the edges of the network (at the sources and destinations) allowing for increased speed and reduced overhead and costs, as well as the possibility for intelligent (and selfish) route or provider choice decisions. The Internet provides some example of such an environment.

\subsection{Wardrop and Nash Equilibria}

The definition of the steady state equilibrium of a traffic network was put forth by J.G. Wardrop in his 1952 treatise [45] which provided two different definitions of traffic assignment concepts. The first is commonly referred to as the Wardrop, or traffic equilibrium, principle and, as we will show later, is a variant of Nash equilibrium for networks. It states that "The journey time on all the routes actually used are equal, and less than those which would be experienced by a single vehicle on any unused route." Wardrop's second principle is what has become known as the system optimum principle, and states that "The average journey time is a minimum." While some point to the economist Pigou, who stated analogous principles in his 1920 Economics of Welfare, as the rightful originator of these ideas, they had not been applied to networks, and transportation networks in particular, until Wardrop's seminal work.

Analogous to Wardrop's first principle is the definition of the famous Nash equilibrium defined in J.F. Nash's 1950 doctoral thesis. Expressing it in terms of network flows, a flow pattern is in Nash equilibrium if no individual decision maker on the network can change to a less costly strategy, or, route. In other words, the Nash equilibrium does not state what can or cannot happen when more than one decision maker changes their strategy (route) simultaneously. When the decision makers in a Nash game are discrete and finite in number, a Nash equilibrium can be achieved without the costs of all used routes being equal, contrary to Wardrop's equilibrium principle. In some cases, Wardrop's principle represents a limiting case of the Nash equilibrium principle, as the number of users becomes very large. We will present some such precise statements on this relationship later in this paper.

$\mathrm{RR} \mathrm{n}^{\circ} 4632$ 
The second Wardrop principle, that of system optimality, assumes that congested networks can be globally optimized. While this can be true for a network which is entirely controlled by a single operator, it is not so with networks of road traffic or with disaggregated telecommunication networks. Therefore, in the remainder of this paper, we will focus on equilibria, rather than pure system optima.

\section{$1.2 \quad$ Equilibria in networks}

Given the fundamental nature of equilibria in many large-scale systems, it is of no surprise that researchers studying transportation networks have been preoccupied with developing models that reproduce this equilibrium, as a function of network characteristics and user demand levels. Typically, transport equilibrium models consider vehicles to be the fundamental units seeking an equilibrium, or, in the case of public transport, the individual traveler. In both of these cases, since the number of users is generally very large, the Wardrop concept, that treats individual user contributions to the costs as infinitesimal, is preferred to the (in this respect, more general) Nash paradigm.

In the context of telecommunication networks, the Wardrop equilibrium is used most often to model the situation in which the routed entities are packets, and routing decisions are taken at the nodes of the networks (rather than by the users) so as to minimize the (per-packet) delay. In many actual networks, the routers at the nodes seek to minimize the per-packet delay in terms of the number of "hops," or nodes, to the destination. There are, however, situations in which it is more advantageous to work with actual delays as cost metrics, rather than the number of hops (see $[10,17]$ ), and it is in these cases that the Wardrop equilibrium has been used to describe the resulting flow patterns. This is the case, for example, in ad-hoc networks in which both users as well as base stations are mobile, or where there are no base stations so that users are responsible to relay messages of other users. For these type of networks a Wardrop type equilibrium has been advocated in [10].

Wardrop equilibria have also been used in telecommunication networks to model a large number of users that can determine individually their route and in which the routed object is a whole session, see [25] (whose model includes in addition some side constraints on the quality of service).

A third context in which Wardrop equilibrium has been used outside of transportation is in distributed computer networks, in which the routed objects are jobs. An individual job can be processed in any of several interconnected nodes (computers) and the routing decision is taken so as to minimize its expected delay in the system (composed of both communication as well as processing delay). Much material on that application can be found in [21].

The most widely studied concept of equilibrium in telecommunication networks is, however, the Nash equilibrium applied to the case of finitely many decision makers. The decision makers typically represent service providers, each of whom can determine how to route the flow generated by its subscribers (and how to split flow between actual paths).

The paper is structured as follows. In Section 2.1, we describe the links between Wardrop and Nash equilibria and the classes of potential and population games. Since these sets are similar but not not equivalent, we hope that the description in this paper encourages transfers of results from one class of games to another. Section 2.3 discusses commodity-link flow models, which, along with the models of section 2.4, are described by variational inequalities. Since the latter class of problems 
has been studied extensively in the transportation equilibrium literature, many of the developments could potentially be applied to models of telecommunication networks. In Section 2.5, we present a few non-additive cost equilibrium models; algorithmic work for this class of models could therefore serve both the transportation and telecom communities.

In Section 3, we present two forms of network pricing. First, in 3.1, we describe models in which link prices are given by Lagrange multipliers, and as such are a form of marginal cost pricing, including recent extensions to that type of pricing that allow auxiliary objectives, such as revenue maximization, to be taken into account, albeit to a limited extent. We then present in 3.2 the more general, bilevel, network pricing problem, which allows operator revenue to be fully maximized, and discuss some algorithmic approaches to solving that very complex problem.

\section{Equilibrium models spanning transport and telecom}

\subsection{Basic Wardrop model}

The basic equilibrium model in traffic networks falls into the category of potential games with an infinite number of users. Indeed, the Wardrop equilibrium condition (the first Wardrop principle, stated above), can be expressed mathematically to state that the flow on every route $r$ serving a commodity, or origin-destination (OD) pair, $w$, is either zero, or its cost is equal to the minimum cost on that OD pair. Along with the fact that the cost on any route serving an OD pair is at least as high as the minimum cost on that OD pair, and the satisfaction of demand for each OD pair, we obtain the following system:

$$
\begin{aligned}
h_{w r}\left(c_{w r}-\pi_{w}\right) & =0, r \in R_{w}, w \in W, \\
c_{w r}-\pi_{w} & \geq 0, r \in R_{w}, w \in W, \\
\sum_{r \in R_{w}} h_{w r} & =d_{w}, w \in W
\end{aligned}
$$

where $h_{w r}$ is the flow on route $r \in R_{w}$, the set of routes joining node pair $w \in W$, the set of origindestination node-node pairs. The cost or delay on that route is $c_{w r}$, and $\pi_{w}$ is the minimum cost on any route joining node pair $w$. The demand for service between the node pair $w$ is denoted $d_{w}$. Define the graph as $G=(\mathcal{N}, \mathcal{A})$. Then, adding non-negativity restrictions $h_{w r} \geq 0$ and $\pi_{w} \geq 0$, the resulting system of equalities and inequalities can be seen as the Karush-Kuhn-Tucker (KKT) optimality conditions of the following optimization problem, known as the Beckmann transformation[7].

$$
\min f(x)=\sum_{l \in A} \int_{0}^{x_{l}} t_{l}\left(x_{l}\right) d x=\sum_{l \in A} \int_{0}^{\sum_{i \in N} x_{i l}} t_{l}(x) d x
$$

subject to

$$
\begin{aligned}
\sum_{r \in R_{w}} h_{w r} & =d_{w}, w \in W, \\
\sum_{w \in W} \sum_{r \in R_{w}} h_{w r} \delta_{w r}^{l} & =x_{l}, l \in A, \\
x_{l} & \geq 0, l \in A,
\end{aligned}
$$


where $x_{i l}$ is the flow of users from OD pair $i$ on link $l, x_{l}$ is $\sum_{i \in N} x_{i l}$ and $\delta_{w r}^{l}$ is a $0-1$ indicator function that takes the value 1 when link $l$ is present on route $r \in R_{w}$.

\subsection{Wardrop equilibrium and Potential games}

The fact that Wardrop equilibrium can be obtained using an equivalent optimization problem with a single player having some cost $f(x)$ is a feature common to a whole class of games known as potential games. This class of games was formally introduced by [32] for the case of finitely many players. It was extended in [42] to the case of population games, which includes the setting of Wardrop equilibrium.

In developing the concept of potential games, game theorists seem not to have been aware of the huge literature on road traffic equilibria starting from Wardrop and Beckmann [45, 7]. Monderer and Shapley write in [32]: "To our knowledge, the first to use potential functions for games in strategic form was Rosenthal (1973)". Interestingly enough, this reference [39] (see also [40]) includes a discrete version of Wardrop equilibrium with finitely many players, called "congestion games".

The original definition of a potential for a game is as follows. Introduce the following $N$-player game $G=\left(N ;\left(S^{i}\right)_{i \in N} ;\left(\eta^{i}\right)_{i \in N}\right)$ where $S^{i}$ is the action set of player $i, S=\times_{i \in N} S^{i}$ and $\eta^{i}(s)$ is the payoff for player $i$ when the multistrategy $s \in S$ is used. For $s \in S$, let $\left(s \mid t^{i}\right)$ denote the multistrategy in which player $i$ uses $t^{i}$ instead of $s^{i}$ and other players $j \neq i$ use $s^{j}$. A potential for the game is defined [32] as a real valued function $P$ on $S$ s.t. for each $i$, every $s \in S$ and every $t^{i} \in S^{i}, P\left(s \mid t^{i}\right)-P(s)=\eta^{i}\left(s \mid t^{i}\right)-\eta^{i}(s)$. Existence and uniqueness of equilibria of potential games in that setting has been established in [32] and [33] ${ }^{1}$.

An adaptation of this definition is needed for population games, see [35, Chap 3] and [42], in which there are $N$ classes of populations of "infinitesimal" players, where the "mass" of players of type $i$ is given by some constants $d_{i}$. Let $\alpha(j, t)$ be the fraction of members of population type $j$ that use action $t \in S^{i}$. A multistrategy is the collection $\alpha=(\alpha(j, t))$. We assume that the payoff $\eta^{i}$ for a player of class $i$ is a function of his own action as well as of the multistrategy $\alpha$. Let $S^{i}$ be the set of actions available to a player of population $i, i=1, \ldots, N$. We say that $\alpha^{*}$ is an equilibrium if for any $i$, any $s \in S^{i}$ and any $t \in S^{i}$ such that $\alpha^{*}(i, t)>0, \eta^{i}\left(t ; \alpha^{*}\right) \geq \eta^{i}\left(s ; \alpha^{*}\right)$. Equivalently, letting $f=-\eta$, we say that flow $\alpha^{*}$ is in equilibrium if the following variational inequality problem (VIP) holds for all $\alpha \in S: f\left(\alpha^{*}\right)\left(\alpha-\alpha^{*}\right) \geq 0$.

We then define $P$ to be a potential for the population game if for each $s$, the vector of payoffs $\eta(s)$ is the gradient of $P(s)$. Under mild conditions on the payoff functions and strategy sets, one can thus establish the existence and uniqueness of equilibria in potential population games. (See [35, Chap 3].)

\subsection{Commodity-link-variable models}

In telecommunication network models, the variable of interest is often the commodity-link flow, $x_{i l}$, rather than the total link flow, $x_{l}$. In this section, we consider a typical model based on commodity-

\footnotetext{
${ }^{1}$ Uniqueness is in fact established among the class of correlated equilibria, of which Nash equilibria is a subset.
} 
link flows as the decision variable, and through it, derive some relations between Nash and Wardrop equilibria.

We have seen that the Wardrop equilibrium can be computed using an equivalent convex optimization problem (related to the potential). We show below that reminiscence of this potential appears also in problems of Nash equilibria with finitely many users: all equilibria satisfying some conditions on the commodity-link flow (condition (11) below) have the same total link flow that can be obtained from some convex optimization problem.

In the network routing problem, the decision variables $x_{i l}$ are restricted by the non-negativity constraints for each link $l$ and player $i: x_{i l} \geq 0$ and by the conservation constraints for each player $i$ and each node $v$ :

$$
r_{v}^{i}+\sum_{j \in \operatorname{In}(v)} x_{i j}=\sum_{j \in O u t(v)} x_{i j}
$$

where $r_{v}^{i}=d_{i}$ if $v$ is the source node for player $i, r_{v}^{i}=-d_{i}$ if $v$ is its destination node, and $r_{v}^{i}=0$ otherwise; $\operatorname{In}(v)$ and $\operatorname{Out}(v)$ are respectively all ingoing and outgoing links of node $v . \quad\left(d_{i}\right.$ is the total demand of player $i$ ).

A typical commodity-link cost function, for the $i$ th commodity on link $l$, is given by

$$
f_{i l}\left(x_{i l}\right)=x_{i l} t_{l}\left(\sum_{j \in \mathcal{I}_{l}} x_{j l}\right),
$$

where $\mathcal{I}_{l}$ is the set of commodities (players) using link $l$. Note that this model resembles the system optimal model defined in Wardrop's second principle. The Lagrangian with respect to the conservation of flow constraints is

$$
L_{i}=\sum_{l \in \mathcal{A}} x_{i l} t_{l}\left(\sum_{j \in \mathcal{I}_{l}} x_{i l}\right)+\sum_{v \in \mathcal{N}} \pi_{i, v}\left(r_{v}^{i}+\sum_{j \in \operatorname{In}(v)} x_{i j}-\sum_{j \in O u t(v)} x_{i j}\right),
$$

for each player $i$. Thus a vector $x$ with nonnegative components satisfying (7) for all $i$ and $v$ is an equilibrium if and only if the following Karush-Kuhn-Tucker (KKT) condition holds:

Let $x_{u v}=\sum_{i \in \mathcal{A}} x_{i, u v}$, where $x_{i, u v}$ is the flow of users from OD pair $i$ on the link defined by node pair $u, v$. There exist Lagrange multipliers $\pi_{i, u}$ for all nodes $u$ and all players, $i$, such that for each pair of nodes $u, v$ connected by a directed link $(u, v)$,

$$
t_{u v}\left(x_{u v}\right)+x_{i, u v} \frac{\partial t_{u v}\left(x_{u v}\right)}{\partial x_{u v}} \geq \pi_{i, u}-\pi_{i, v}
$$

with equality if $x_{i, u v}>0$.

Define $\pi_{u}=\sum_{i} \pi_{i, u}$. Taking the sum over all players we get the following necessary conditions for $x$ to be an equilibrium for each link $(u, v)$ :

$$
I t_{u v}\left(x_{u v}\right)+x_{u v} \frac{\partial t_{u v}\left(x_{u v}\right)}{\partial x_{u v}} \geq \pi_{u}-\pi_{v}
$$

with equality if $x_{i, u v}>0$ for all $i$, where $I$ is the total number of players. 
Assume that all players have the same source and destination, and let $d$ be the sum of commodity demands. Then (9) are the KKT conditions for optimality of the vector $\left\{x_{l}\right\}$ with nonnegative components satisfying the conservation of flow constraints in the routing problem (single commodity) where the cost to be minimized is given by

$$
\sum_{l \in A} x_{l} t_{l}\left(x_{l}\right)+(I-1) \int_{0}^{x_{l}} t_{l}(y) d y
$$

and where the total demand to be shipped from the common source to the common destination is $d$; in particular, (9) holds with equality if $x_{u v}>0$. Assume further that $t_{l}$ are strictly convex, or more generally that expression (10) is strictly convex. Then this problem has a unique solution in total link flows, which we denote $\left(x_{l}^{*}\right)$.

Now, let $\left\{x_{i l}\right\}$ be a Nash equilibrium for the original problem having costs (8) with the property:

$$
\text { A1. Whenever } x_{i l}>0 \text { for some } i \text { and } l \text { then } x_{j l}>0 \text { for all players } j \text {. }
$$

A1 describes a property of the equilibrium: if (at equilibrium) one player sends positive flow through a link, then so do all other players. Under assumption $\mathrm{A} 1$, it follows that for all $l, \sum_{i} x_{i l}=x_{l}^{*}$. Note however, that this is not true in general if A1 does not hold, since $x_{l}^{*}$ need not be expressible as the sum over $I$ of some nonnegative $x_{i l}$ that satisfy (7).

Remark The above is an alternative proof to the one in [34] of the uniqueness of the total link flows at all Nash equilibria satisfying A1.

Taking the limit in (10) as the number of players $I \rightarrow \infty$, the second term in (10) dominates, and by continuity of the functions and compactness of the feasible set, we observe that both the objective function and the solution approach that of the Wardrop equilibrium.

\subsection{General models and variational inequalities}

The basic equilibrium model imposes a number of simplifications on the model of the traffic flow phenomenon, and in particular, on the travel time, or impedance, functions.

Most notably, for the potential function to exist, the travel time function, $t_{l}$, defined for each link of the network, $l \in A$, must be integrable. The most common way for this to occur is that the travel time on a link $l$ depends only upon the flow present on the link $l$, that is, $t_{l}(x)=t_{l}\left(x_{l}\right)$. This simplification, in the traffic context, means that interactions between different traffic streams at junctions cannot be modeled within this paradigm (even if we use a virtual link to model the node), since then, the travel time on a link $l$ that reaches the junction is a function of flows on some or all links meeting link $l$ at the junction, that is, $t_{l}(x)=t_{l}\left(x_{1}, \ldots, x_{l}, \ldots, x_{m}\right)$ (and is not just a function of the sum of flows). The simplification also imposes that only a single class of users is modeled, since multiple classes of users would interact on each link, resulting once again in multivariate link travel time functions. In short, when the identities (in terms of multiple user classes, or the multiple links they use) is needed in the cost function of a single link, then the single-class model is no longer applicable.

When the link travel time functions are multivariate, it is usually the case that no potential that can be obtained by integrating the travel time functions as in the basic model. Examples of 
multivariate link cost functions can be found in the literature on modeling signalizes junctions on a road network [18]. Other examples can be found in the modeling of multimodal networks, such as networks on which buses and cars share the road space or trucks and light vehicles, since each traffic class effects the traffic differently, and each class has its own travel time function, depending on all classes present on the link. Some characteristics of this type of multivariate cost functions can be found in [44].

Although the potential function approach cannot be used to describe the multivariate equilibrium, the Wardrop equilibrium conditions are valid regardless of whether the cost functions are univariate or multivariate, and they can be expressed for both types of cost functions in a compact variational framework.

In telecommunication network planning, link impedance functions can often be quite complex, due to the underlying probabilistic phenomena as well as the interacting cost components of delay, packet loss, jitter, etc... A typical form of the commodity-link cost functions (see, for example, [34]) is

$$
t_{i l}\left(x_{i l}, x_{l}\right)=x_{i l} t_{l}\left(x_{l}\right)=\frac{x_{i l}}{C_{l}-x_{l}},
$$

where, as before, $x_{l}=\sum_{i=1 . . I} x_{i l}$ is the flow of all classes $i$ on link $l$. The constant $C_{l}$ is the capacity of link $l$. The user classes in this case correspond to commodities, or origin-destination demands. For more justification on this type of delay models, see [5, 22]. In some simple settings, such as a network of parallel arcs, when the sum of the demands of all classes is less than link capacity, or on some one-commodity networks, the authors in [34] show uniqueness of the Nash equilibrium. Also under "diagonal strict convexity", the authors show uniqueness of the Nash equilibrium, yet this condition only holds under quite restrictive conditions on the cost functions or on the topology [1]. In $[34,3]$, uniqueness of the link class flows is shown for costs of the form (12) for general networks under assumption $\mathrm{A} 1$ (see eq. (11).

We can analyze the Wardrop equilibrium for this system easily by expressing it as the solution $\left\{x_{i}^{*}\right\}$ of the following variational inequality:

$$
t_{i}\left(x^{*}\right)^{T}\left(x_{i}^{*}-x_{i}\right) \leq 0,
$$

for all feasible class-flow vectors, $x_{i}$, where, as above, the vector $x=\sum_{i=1 . . I} x_{i}$ and $t_{i}=t$ for all classes $i=1 . . I$. Since the Jacobian of the mapping $t$ is clearly singular $\left(\partial t_{i}(x) / \partial x_{j}=\partial t_{j}(x) / \partial x_{i}\right.$ for all $i, j$ ), we recover the nonuniqueness of the equilibrium (in the variable $x_{i}$ ) that was observed by [34] to occur on general networks.

We can also contrast the Wardrop and Nash equilibria through this example. The Nash equilibrium $x_{i}^{*}$ satisfies

$$
\sum_{l \in R_{i}} x_{i l}^{*} t\left(x_{i l}^{*}, x_{\neq i l}^{*}\right) \leq \sum_{l \in R_{i}} x_{i l} t\left(x_{i l}, x_{\neq i l}^{*}\right)
$$

for each user class $i=1 . . I$, where the index $\neq i$ includes all classes not equal to $i$. Rewriting, we obtain that $\sum_{l \in R_{i}} x_{i l}^{*} t\left(x_{i l}^{*}, x_{\neq i l}^{*}\right)-\sum_{l \in R_{i}} x_{i l} t\left(x_{i l}, x_{\neq i l}^{*}\right) \leq 0$ for each $i \in I$ and therefore when

$$
t\left(x_{i l}^{*}, x_{\neq i l}^{*}\right)-t\left(x_{i l}, x_{\neq i l}^{*}\right)=0
$$

the above reduces to $\sum_{l \in R_{i}}\left(x_{i l}^{*}-x_{i l}\right) t\left(x_{i l}^{*}, x_{\neq i l}^{*}\right) \leq 0$, for each $i \in I$, which is equivalent to the (Wardrop) VIP with cost operator $t$ and classes given by users $i \in I$. Indeed, (14) occurs precisely when the influence of an additional user on the cost, $t$, is 0 . 
Another example of general costs in telecommunications can be found in [2], where the network model includes dropping of calls if capacity is exceeded. The cost criterion for each user class $i$ is the probability that his message is rejected along its path, given by

$$
B_{i}(x)=1-\frac{\sum_{\mathbf{m} \in S_{i}} \prod_{j=1}^{I}\left(x_{j}^{m_{j}} / m_{j} !\right)}{\sum_{\mathbf{m} \in S} \prod_{j=1}^{I}\left(x_{j}^{m_{j}} / m_{j} !\right)} .
$$

$S$ is the set of feasible "states"; $S_{i}$ is the set of states for which another call of user $i$ can still be accepted (without violating capacity constraints), $\mathbf{m}$ is the system state whose $j$ th component $m_{j}$ is the number of class $j$ calls in the system. Hence the number of terms in the cost function depends upon the configuration and capacity constraints of the network. (The larger the number of feasible paths for a user class, the more terms present.) When each class, which can be represented by an origin-destination pair, seeks a Wardrop equilibrium, the corresponding variational inequality is to find $x_{i}^{*}$ such that $B_{i}\left(x^{*}\right)^{T}\left(x_{i}^{*}-x_{i}\right) \leq 0$, for all $x_{i}$, for all classes $i$. Even in the simplest topology of parallel links, it has been shown in [2] that there may exist several equilibria with different total link flows.

Depending on the form of monotonicity satisfied by the cost operator in the variational inequality, one can choose convergent algorithms for its solution, as well as determine whether or not the solution will be unique. Weaker forms of monotonicity for which the mathematical properties and a number of convergent algorithms are known include pseudo-monotonicity and strong nested monotonicity. (See $[31,13]$ for the latter.)

\subsection{Additive versus non-additive models}

The most widely studied performance measure investigated to date in transportation, computer and telecommunication networks has been the expected delay. In transportation networks, this cost metric leads naturally to models in which the route costs are additive functions of link costs along the route, that is $c_{r}(x)=\sum_{l \in R} t_{l}\left(x_{l}\right)$.

In telecommunication networks, exogenous arrivals of jobs or of packets are modeled as Poisson processes. Delays at links are modeled by infinite buffer queues with i.i.d. service times, independent of the interarrival times. In the particular case in which the service time at a queue is exponentially distributed, then whenever the input process has a Poisson distribution so will the output stream. This makes the modeling of service times through exponential distributions quite appealing, and makes the cost along a path of tandem queues additive. However, the expected delay turns out to be additive over constituent links in a general topology under a much more general setting, known as BCMP networks (named after its authors [5]).

Indeed, as long as the exogenous arrivals have Poisson distributions and under fairly general assumptions on the service order and service distribution, the expected delay over each link is given by the expected service time divided by $(1-\rho)$, where $\rho$ is the product of total average traffic flow at the queue and the expected service time. This general framework also allows for the modeling of multiclass systems, i.e. where different traffic classes require different expected service times at a queue, see [22].

Other more general types of separable additive cost functions have been used in telecommunication networks which can represent physical link costs due to congestion pricing, see e.g. [34]. 
However, in both transportation and telecommunication networks, equilibrium models in which path costs are not the sum of link costs do arise.

In the transport sector, when environmental concerns are taken into account, such as the pollution associated with trips, non-additive terms arise. Path costs due to tolls or public transport costs are generally non-additive as well, since they are calculated over entire paths, and cannot be decomposed into a sum of the costs on component links.

In $[15,9]$, the authors discussed properties of a bicriteria equilibrium problem in which both time delay and prices are modeled on the links. Non-additivity arises from the nonlinear valuation of the tradeoff between time and money, known as a nonlinear value of time. For example, users are willing to pay more (or less) per minute for longer trips than for shorter trips. That is, a route cost function may be expressed as $c_{r}(x, p)=V\left(\sum_{l \in R_{i}} t_{l}\left(x_{l}\right)\right)+\sum_{l \in R_{i}} p_{l}$, where $V: \Re_{+}^{m} \mapsto \Re_{+}$is the nonlinear value of time function.

In telecommunication networks, many important performance measures are neither additive nor separable. The first example is that of loss probabilities when the network contains finite buffer queues. We note that in this case there is no flow conservation at the nodes ${ }^{2}$. This model has been dealt with by light traffic approximations which are additive and separable [14, 20].

Another performance measure (already mentioned in the previous subsection) that has been studied in the context of network equilibrium is that of rejection probabilities. The network consists of resources at each link, and requests for connections between a source and a destination. The resources are limited, and a connection can only be established if there are sufficiently many resources along each link of a route between the source and destination. For the case where connections arrive according to a Poisson process and where calls last for an exponentially distributed duration (these assumptions model telephone networks well), simple expressions for rejection probabilities (i.e. the probability that an arriving call will find the line busy) are available. These expressions are neither separable nor additive. Such networks have been studied in $[2,6]$.

When route costs are no longer the sum of constituent link costs, algorithms for solving the network equilibrium problems must be modified; indeed, underlying most algorithms for the network equilibrium problem is a shortest path search, and standard searches all suppose additive path costs. One algorithm for the non-additive route cost model can be found in [16]; another can be found in [38].

\section{Pricing}

The pricing of network services, in transportation and telecommunications, has taken on more importance in the past decade. In transportation networks, congestion pricing, such as road tolls which adapt to time of day, and could, in principle, vary with the congestion level, have become a reality in many parts of the world. Similarly, pricing of communications networks and the Internet have become intense areas of research activity since telecom deregulation, and due to the development of Internet services, such as tele-conferencing, software leasing, and e-commerce.

\footnotetext{
${ }^{2}$ Note that flow conservation fails even in the case of infinite queues when one considers multicast applications in which packets are duplicated at some nodes, see [12].
} 


\subsection{Marginal cost pricing}

The vast majority of the literature on pricing of networks supposes that the pricing scheme is based upon shadow prices, or marginal costs. That is, the marginal cost of adding a new request, or user, to the network defines the price that should be charged to that user. (See [35] for a list of references from the transportation literature, and $[23,24,29]$ in the telecommunications literature.)

When there are no capacity constraints in the model, the marginal cost of adding a new user on link $a \in A$ within the Wardrop equilibrium model is given by

$$
t_{a}\left(x_{a}\right)+x_{a} t_{a}^{\prime}\left(x_{a}\right)
$$

The difference then between marginal costs and user costs is the second term, $x_{a} t_{a}^{\prime}\left(x_{a}\right)$. For the user equilibrium solution to be "driven" to the system optimal solution, the cost of adding each new user must be taken into account, such as through marginal cost pricing. This means substituting delay function (15) for $t_{a}\left(x_{a}\right)$ in the Wardrop equilibrium model.

In telecommunication networks, capacity constraints are generally present on each link in the model. The Lagrange multipliers on those constraints provide another marginal cost, representing an additional cost that can be imposed on each user that would exceed the capacity on the link. By construction, the multipliers on those constraints, and therefore the link prices, are zero unless the link is at capacity. (Note that these prices need not, however, be unique.) This price can be understood as a control measure, a "cost" imposed in addition to the travel cost itself that keeps the flow within the boundaries of the capacity constraints, without actually having to enforce the capacity constraints, see e.g. [28].

In the telecommunications literature, this notion of pricing is referred to as the Proportional Fairness principle, when the network flow problem is defined by the maximization of the sum of logarithmic user's utility functions $[23,24]$. That is, the network flow objective is given by $\max _{d \in D} \sum_{i \in I} \log \left(d_{i}\right)$ subject to flow conservation, link capacity, and non-negativity constraints, defined as the set $D$ of feasible resource allocations, where $d \in \Re_{+}^{W}$ is the amount of bandwidth to be allocated to user $i \in I$, defined by an origin-destination node pair. The objective of the proportional fairness bandwidth allocation and pricing scheme is therefore to determine the origin-destination demand level to allocate to each pair that maximizes a logarithmic utility function, and satisfies network constraints.

In $[27,26]$, the authors showed that the non-uniqueness of the Lagrange multipliers in capacityconstrained equilibrium problems could be used to optimize traffic management schemes by defining a secondary function over the set of multipliers. In [31, 11], this technique was applied to the Proportional Fairness pricing model so as to permit revenue maximization using fair prices. That is, the network operator would solve $\max _{\lambda \in \Lambda\left(x^{*}\right)} \lambda^{T} x^{*}$, where $x^{*}$ is a proportionally fair resource allocation and $\Lambda\left(x^{*}\right)$ is the (polyhedral) set of Lagrange multipliers at $x^{*}$. This approach has been studied and algorithms developed in [26, 41]. In [11] numerical experience aimed at studying the degree to which revenue maximization is possible while still maintaining the properties of the marginal cost pricing scheme is provided. 


\subsection{Pricing strategies with other objectives}

While Lagrange multiplier-based pricing strategies have a historical foundation, in that they quantify the notion of marginal cost pricing of networks, they do not take into account fully the desire of the network operator to maximize revenue. Indeed, the link prices are heavily constrained by the fact that they must be Lagrange multipliers, or by the fact that they must precisely drive flows to the system optimal solution. The strategy described above does permit limited profit maximization to be achieved, when the total revenue is not unique, as it may be in some cases (see $[27,11]$ ).

However, in general, for revenue maximization to be realized, prices can no longer be tied to Lagrange multipliers. In the case of capacity constraints, the multipliers, as stated earlier, are only positive when capacity is reached. This alone reduces potential for revenue. Furthermore, in telecommunication networks, there are cases in which further integrality constraints are imposed: one cannot split the flows at any proportion but instead, one has to choose the transmission rate over each link among some given set. In such cases, capacity need not be reached exactly for the network to be congested. (See [19] for a solution to this problem in a different context.)

The general paradigm that models optimal network pricing is known as the bilevel program; the lower level of the problem represents the user equilibrium or network flow problem with prices as a parameter, and the upper-level, or network operator's problem, in $g$, seeks to optimally set the prices given the response of the users or flow pattern, to those prices. The mathematical formulation is given by

$$
\max g(x, p)=x^{T} p, \quad x \in S(p), \quad p \in P
$$

where $S(p)=\left\{x^{*}: F\left(x^{*}, p\right)^{T}\left(x^{*}-x\right) \leq 0, x \in X(p)\right\}, X(p)$ being the set of constraints on flow variables $x$ (network constraints, non-negativity, ... along with any other constraints that may depend on the price vector, $p$.) and where $F=\nabla f$ if the (Nash or Wardrop) equilibrium problem admits an equivalent convex optimization formulation. Recall that $f$ are the cost functions of the players in the Nash game, or the objective function of the optimization form of the Wardrop equilibrium, in the separable case. The bilevel programming problem is known to be nonconvex and non-differentiable, even in the most favorable circumstances, when the users' equilibrium problem is constrained. It is therefore very difficult to solve over networks, and most methods in the literature are designed to converge to any stationary point of the problem. An exception is the algorithm of [30] which uses a particular decomposition based upon the structure of the induced region, and has been shown experimentally to converge to the global maximum of the problem. The most popular methods for solving the bilevel program to a local optimum include penalty methods, in which the constraint $x \in S(p)$, expressed by its KKT conditions, is penalized in the objective, $g$, as well as sensitivitybased methods, that obtain a subgradient of the objective, $g$, through sensitivity analysis and apply subgradient or bundle methods. (See for the penalty approach [4] and [37] for the sensitivity-based methods.)

\section{Perspectives}

This paper has highlighted a number of areas in which common features between transportation and telecommunication network models exist, and where future research effort would be worthwhile. Here we summarize a few of the areas that seem particularly worthwhile for further research effort. 


\subsection{Different definitions of equilibrium and games}

It is clear that through lack of a common forum, much work on network games is duplicated in different communities, often with the strength of the results varying across the board. We discussed some examples of this in Section 2.2, mentioning in particular that Wardrop equilibrium is a special case of a potential game, which is in turn a special case of population games. On the one hand, it is worth studying whether any results on population games generalize those known for Wardrop equilibria, or vice-versa. Most work on potential games considers a finite number of players, and so appear less useful in transportation networks than in telecommunications settings. We note that some work in discrete Wardrop equilibria can be found in [8], and some extensions of potential games to infinite many players can be found in [42].

\subsection{General costs}

We presented several settings in which costs are more general than the standard separable, total link flow cost functions so prevalent in the transportation literature. In particular, telecommunication models generally make use of commodity-link flow variables. While this can be seen as a special case of the multi-class models found in the transportation literature, we saw that through the particular form of the commodity-link costs, such as (12), a number of much stronger properties can be obtained. The most surprising of these is that the commodity-link flows can in some cases be unique (e.g., for networks of parallel links, ...). While this model and the uniqueness result does not immediately transfer to transportation networks, since the costs there will generally not satisfy the properties assumed, one could consider looking at particular networks and cost forms in transportation models to deduce much stronger properties than have been assumed to hold.

Similarly, by formulating more general models from telecommunications as variational inequalities, it is possible to determine their uniqueness properties over general networks quite readily, and, especially, to propose a much wider range of algorithms for solving for those equilibria.

Another non-standard form of equilibrium models are those which do not have additive costs over constituent links of the paths. Some work on these models exists in both communities, and we have cited a few references on such work. It seems clear that there should be considerable possibilities for adapting the results mentioned to the applications of both transport and telecom models.

\subsection{Pricing}

We have provided quite a cursory description of optimal network pricing, since a large body of literature on this topic exists in the telecommunication setting. However, the vast majority of that work does not include equilibrium models, or does not appear to be readily transferable to transportation modeling. We have therefore chosen not to include those references in this paper. We do, however, describe a well-known approach proposed for telecommunications pricing that can be shown to be a special case of an approach proposed for transportation system management. The paradigm of bilevel programming seems particularly appropriate to both telecommunications and transportation pricing, when marginal costs are not sought. Adapting this paradigm to particular settings and applications will likely become a very active field of research. However, the algorithmic 
issues that will result are considerable; attention could be focused on devising more rapid and decomposable approximation algorithms.

\section{References}

[1] E. Altman, T. Başar, T. Jiménez and N. Shimkin, "Competitive routing in networks with polynomial cost", IEEE Trans. on Automatic Control, Vol 47, pp. 92-96, Jan. 2002.

[2] E. Altman, R. El-Azouzi, and V. Abramov, "Non-cooperative routing in loss networks", Performance Evaluation, Vol 49, Issue 1-4, pp. 43-55, 2002.

[3] E. Altman and H. Kameda, "Equilibria for Multiclass Routing Problems in Multi-Agent Networks", 40th IEEE Conference on Decision and Control, Orlando, Florida, U.S.A., Dec. 2001.

[4] J.F. Bard, 'Practical Bilevel Optimization: Algorithms and Applications" Kluwer Book Series: Nonconvex Optimization and its Applications, 30, 1998.

[5] F. Baskett, K.M. Chandy, R.R. Muntz and F. Palacios, "Open, closed, and mixed networks of queues with different classes of customers," J. ACM 22, 248-260, 1975.

[6] Bean, N.G., Kelly, F.P. and Taylor, P.G. (1997) Braess's paradox in a loss network. J. Appl. Prob., 34, 155-159.

[7] M. Beckmann, C. B. McGuire and C. B. Winsten, Studies in the Economics of Transportation, New Haven: Yale Univ. Press, 1956.

[8] D.H. Bernstein, "Programmability of continuous and discrete network equilibria", $\mathrm{PhD}$ thesis, University of Pennsylvania, 1990.

[9] D.H. Bernstein and L. Wynter, 'Issues of Uniqueness and Convexity in Non-additive Bi-criteria Equilibrium Models", Proceedings of the EURO Working Group on Transportation, Rome, 2000.

[10] D. Bertsekas and R. Gallager, Data Networks, Prentice Hall, Englewood Cliffs, New Jersey, 1987.

[11] M. Bouhtou, M. Diallo, and L. Wynter, "A numerical study of Lagrange-multiplier-based pricing schemes", to appear in Optimization and Optimal Control, P.M. Pardalos, I. Tsevendorj, and R. Enkhbat, Eds. World Scientific Publishing Co., 2003.

[12] T. Boulogne, E. Altman, "Competitive Routing in Multicast Communications", submitted, 2001. Available at http://www-sop.inria.fr/mistral/personnel/Thomas.Boulogne/

[13] G. Cohen and F. Chaplais, "Nested Monotony for Variational Inequalities over Product of Spaces and Convergence of Iterative Algorithms" Journal of Optimization Theory and Applications, $\mathbf{5 9}$ (3) 1988.

[14] E. Dinan, D. O. Awduche and B. Jabbarie, "Optimal traffic partitioning in MPLS Networks", Proceedings of IFIP-TC6 Networking, Paris, France, May 14-19, 2000.

[15] S. A. Gabriel and D. Bernstein, "The Traffic Equilibrium Problem with Nonadditive Costs," Transportation Science, 31, 337-348, 1997. 
[16] S. A. Gabriel and D. Bernstein, 'Nonadditive Shortest Paths: Subproblems in Multi-Agent Competitive Network Models," Computational and Mathematical Organization Theory, 6, 2945,2000 .

[17] P. Gupta and P. R. Kumar, "A system and traffic dependent adaptive routing algorithm for ad hoc networks," Proceedings of the 36th IEEE Conference on Decision and Control, pp. 23752380, San Diego, Dec. 1997.

[18] B.G. Heydecker, "Some consequences of detailed junction modeling in road traffic assignment" Transportation Science, 17, 263-281, 1983.

[19] F. Jarray and L. Wynter, "An Optimal Smart Market Auction for the Pricing of Telecommunications Services", INRIA Research Report 4310, 2002. Available at http://www.inria.fr/rrrt/index.en.html

[20] T. Jiménez, "A competitive routing approach for packet loss minimization", manuscript.

[21] H. Kameda, J. Li, C. Kim and Y. Zhang, Optimal Load Balancing in Distributed Computer Systems, Springer, 1997.

[22] H. Kameda and Y. Zhang, "Uniqueness of the solution for optimal static routing in open BCMP queueing networks," Math. Comput. Modelling 22(10-12), 119-130, 1995.

[23] F. Kelly, "Charging and Rate Control for Elastic Traffic," European Trans. Telecommunications, 8, 33-37, 1997.

[24] F. Kelly, A. K. Maulloo, and D. K. H. Tan, 'Rate Control for Communication Networks: Shadow Prices, Proportional Fairness, and Stability," Journal of the Operational Research Society, 49, 237-252, 1998.

[25] Y. A. Korilis and A. Orda, 'Incentive-Compatible Pricing Strategies for QoS Routing", in Proceedings of IEEE INFOCOM'99, New York, NY, USA, March 1999

[26] T. Larsson, J. Lundgren, M. Patriksson, and C. Rydergren, "Current Trends in Transportation and Network Analysis - papers in honor of Michael Florian", Michel Gendreau, Patrice Marcotte, Eds., 147-164, Kluwer Applied Optimization Series, 63, 2002.

[27] T. Larsson and M. Patriksson, 'Side Constrained Traffic Equilibrium Models-Traffic Management Through Link Tolls", in Equilibrium and Advanced Transportation Modelling, P. Marcotte and S. Nguyen (eds.), Kluwer Academic Publishers, Boston, MA, pp. 125-151, 1998.

[28] T. Larsson and M. Patriksson, "Side constrained traffic equilibrium models: analysis, computation and applications", Transportation Research, Part B, Vol 33, pp. 233-264, 1999.

[29] S.H. Low and D.E. Lapsley, "Optimization flow control I: Basic algorithm and convergence," IEEE/ACM Transactions on Networking, 7, 961-874, 1999.

[30] E. Mancinelli, J.-P. Quadrat, and L. Wynter, "A new algorithm for bilevel network pricing", manuscript.

[31] P. Marcotte and L. Wynter, "A new look at the multi-class network equilibrium problem", to appear in Transportation Science.

[32] D. Monderer and L. S. Shapley. Potential games. Games and Econ. Behavior, 14:124-143, 1996. 
[33] A. Neyman, "Correlated equilibrium and potential games", International Journal of Game Theory, 26:223-227,1997.

[34] A. Orda, R. Rom and N. Shimkin, "Competitive routing in multi-user environments," IEEE/ACM Trans. on Networking, 510-521, 1993.

[35] M. Patriksson, The traffic assignment problem: Models and methods, VSP, Utrecht, 1994.

[36] M. Patriksson, Nonlinear programming and variational inequalities: A unified approach, Kluwer Academic Publishers, Dordrecht, 1999.

[37] M. Patriksson, "Sensitivity analysis of traffic equilibria", to appear in Transportation Science, 2003.

[38] M. Patriksson, "Algorithms for computing traffic equilibria", to appear in Networks and Spatial Economics, 2003.

[39] R. W. Rosenthal, "A class of games possessing pure strategy Nash equilibria", Int. J. Game Theory, 2:65-67, 1973.

[40] R. W. Rosenthal, 'The network equilibrium problem in integers", Networks, 3:53-59, 1973.

[41] C. Rydergren, Decision support for strategic traffic management: An optimization-based methodology, PhD dissertation, Department of Mathematics, Linköping University, Sweden, 2001.

[42] W. H. Sandholm. Potential games with continuous player sets. submitted, 2000. See also Evolutionary justification of Nash Equilibrium by the same author, PhD thesis, Northwestern University, 1998.

[43] Y. Sheffi, Urban Transportation Networks, Prentice Hall, Englewood Cliffs, NJ, 1985.

[44] P. Toint and L. Wynter, "Asymmetric traffic assignment, a coherent formulation", Proceedings of the 13th International Symposium on Transportation and Traffic Theory, (ISTTT), Lyon, France, 24-26 July, J.-B. Lesort, Ed., Pergammon, pp. 237-260, 1996.

[45] J. G. Wardrop, "Some theoretical aspects of road traffic research, Engineers, Part II, 1952, pp. $325-378$.

[46] L. Wynter, "Optimizing proportionally fair prices", INRIA Research Report 4311, 2001. Available at http://www.inria.fr/rrrt/index.en.html 


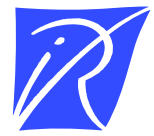

Unité de recherche INRIA Rocquencourt Domaine de Voluceau - Rocquencourt - BP 105 - 78153 Le Chesnay Cedex (France)

Unité de recherche INRIA Lorraine : LORIA, Technopôle de Nancy-Brabois - Campus scientifique 615, rue du Jardin Botanique - BP 101 - 54602 Villers-lès-Nancy Cedex (France)

Unité de recherche INRIA Rennes : IRISA, Campus universitaire de Beaulieu - 35042 Rennes Cedex (France)

Unité de recherche INRIA Rhône-Alpes : 655, avenue de l'Europe - 38330 Montbonnot-St-Martin (France)

Unité de recherche INRIA Sophia Antipolis : 2004, route des Lucioles - BP 93 - 06902 Sophia Antipolis Cedex (France) 